\title{
Jugular Venous Thrombosis, a Rare Location of the Embolic Thrombo Venous Disease about a Case in Guinea
}

\author{
Abdoulaye Camara ${ }^{*}{ }^{(0)}$, Sana Samoura1, Aly Samoura1, Diarra Koivogui1, Djibril Sylla², \\ Mohamed Doumbouya1, Morlaye Soumaoro', Ibrahima Sory Barry¹, Elhadj Yaya Balde1, \\ Mariama Beavogui1, Mamadou Dadhi Balde', Mamady Conde ${ }^{1}$ \\ ${ }^{1}$ Cardiology Service, Ignace Deen CHU, Conakry, Republic of Guinea \\ ${ }^{2}$ Medico-Surgical Emergency Service, National Hospital of Donka, Conakry, Republic of Guinea \\ Email: ^mariboudou@gmail.com
}

How to cite this paper: Camara, A., Samoura, S., Samoura, A., Koivogui, D., Sylla, D., Doumbouya, M., Soumaoro, M., Barry, I.S., Balde, E.Y., Beavogui, M., Balde, M.D. and Conde, M. (2019) Jugular Venous Thrombosis, a Rare Location of the Embolic Thrombo Venous Disease about a Case in Guinea. World Journal of Cardiovascular Diseases, 9, 628-631. https://doi.org/10.4236/wjcd.2019.99055

Received: July 29, 2019

Accepted: August 31, 2019

Published: September 3, 2019

Copyright $\odot 2019$ by author(s) and Scientific Research Publishing Inc. This work is licensed under the Creative Commons Attribution International License (CC BY 4.0).

http://creativecommons.org/licenses/by/4.0/

\begin{abstract}
Observation: This patient was a 40-year-old housewife with dysphonia, physical asthenia, palpitations, fever and cervical tumefaction that had been going on for 2 months, no known cardiovascular risk factor, such as medical history, ischemic stroke. Heart sounds were regular at $110 \mathrm{bpm}$, blood pressure $=120 / 80 \mathrm{mmhg}$, to the lungs there are sibilant rattles. Elsewhere, there is a painful left lateral cervical tumefaction febrile to the touch. Temperature $=$ $38^{\circ} \mathrm{C}$. The rest of the exam is peculiar. Conclusion: Jugular vein thrombosis is a rare variety of unusual localization of venous thromboembolism. It must be suspected in the presence of a painful cervical swelling and confirmed by magnetic resonance imaging or to scan with contrast or ultrasound. Anticoagulant therapy should be instituted as soon as possible to avoid the formidable complication of pulmonary embolism.
\end{abstract}

\section{Keywords}

Jugular Vein Thrombosis, Ignace Deen, Guinea

\section{Introduction}

Thrombosis of the internal jugular vein is very rare in the absence of local promoting factors, including central venous catheterization; hyperhomocysteinemia is currently considered a factor favoring deep vein thrombosis [1]. Deep vein thrombosis (DVT), classically located in the lower limbs, is inseparable from the immediate complication of pulmonary embolism (PE), which justifies the concept of thromboembolic venous disease (DVT). Other locations of DVT, al- 
though rarer, may occur and are typically called deep-seated deep vein thromboses [2].

\section{Observation}

This patient was a 40-year-old housewife with dysphonia, physical asthenia, palpitations, fever and cervical tumefaction (Figure 1) that had been going on for 2 months. As an antecedent ischemic stroke without cardiovascular risk factor. Heart sounds were regular at $110 \mathrm{bpm}$, blood pressure $=120 / 80 \mathrm{mmhg}$, to the lungs there are sibilant rattles. Elsewhere, there is a painful left lateral cervical tumefaction febrile to the touch. Temperature $=38^{\circ} \mathrm{C}$. The rest of the exam is peculiar. Electrocardiogram showed sinus tachycardia. Ultrasonography of the neck resulted in a normal ultrasonic appearance of the thyroid glands, total thrombosis of the right jugular vein associated with ipsilateral lymphadenopathy (Figure 2). Venous doppler echo of the neck concluded with thrombosis of the acute right jugular vein. Cardiac Echodoppler was normal. The biology revealed

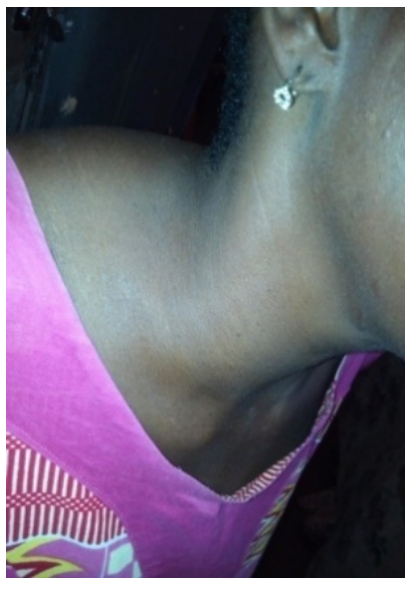

Figure 1. Image showing lateral tumefaction of the neck.

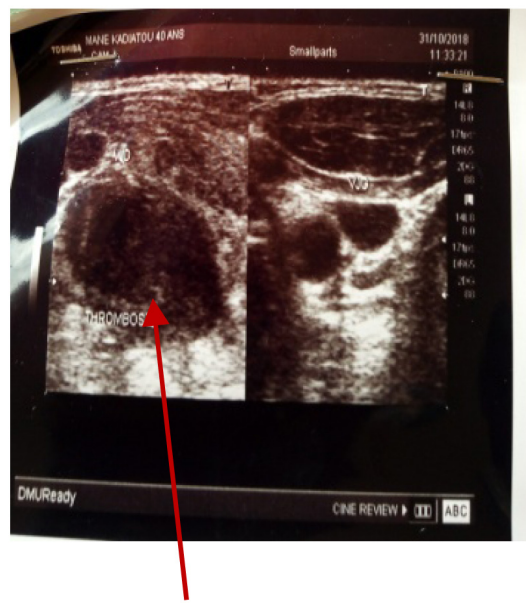

Figure 2. Ultrasound of the neck showing a normal ultrasonic appearance of the thyroid glands, total thrombosis of the right jugular vein associated with ipsilateral lymphadenopathy. 
hypocalcemia at $0.70 \mathrm{mg} / \mathrm{dl}$ (normal between 8.6 and $10.3 \mathrm{mg} / \mathrm{dl}$ ); Toxoplasmosis IgG positive at $497 \mathrm{IU} / \mathrm{ml}$ (normal IgG negative); 1 st INR at 2.66; 2nd INR at 2.70 (normal between 2 and 3); D-dimer = $1000 \mathrm{ng}$ (normal < $500 \mathrm{ng}$ ); Leukocytes at 11 giga/l (normal between 4 and 10 giga/l); Negative SRV.

At the end of the clinical and para-clinical examination the diagnosis of jugular vein thrombosis, hypocalcemia and toxoplasmosis was retained. She had benefited from anti-coagulation based on enoxaparin and previscan, calcium 500 mg. The evolution was marked by the regression of the signs after 4 days of treatment.

\section{Discussion}

We report the case of right jugular vein thrombosis in a Guinean woman.

Venous thromboembolism (VTE) is a common and serious condition with multifactorial pathogenesis. It may be the first manifestation of an underlying pathology that has been previously infra-clinical [3]. All conditions that modify blood flow where coagulation mechanisms may increase the risk of thrombosis. These conditions are often accompanied by risk factors related to the age and morbidities of the patient [4]. This is the case of our patient who perfectly reflects this unusual location of venous thrombosis. In general, the clinical presentation of jugular thrombosis is initially nonspecific and is manifested by unilateral painful swelling on palpation of the vessels, possibly associated with cervical lymphadenopathy. The patient sometimes adopts an "analgesic position", characterized by a lateral flexion of the neck that can lead to torticollis. Various complications can occur, for example, dysphagia, dyspnea, and secondary thrombus infection with subsequent sepsis [5]. Another dreaded complication is pulmonary embolism, with thromboembolic obstruction of the pulmonary arteries. The clinical suspicion of acute thrombosis of the jugular vein must be confirmed as soon as possible by medical imaging: magnetic resonance imaging or to scan with contrast medium or ultrasound [5]. In our case it is the venous doppler echo performed a highlighted a thrombosis of the acute right jugular vein. It has a place of choice in establishing the diagnosis of jugular venous thrombosis (TVJ). The problem is to think about cervical swelling. Laboratory tests should include D-dimers as relatively nonspecific but very sensitive markers of thromboses [5]. In our case the D-dimer was $1000 \mathrm{ng} / \mathrm{l}$. According to Virchow, the etiology of VTE can be summed up in 3 mechanisms: blood stasis favored by any state of slowing of the circulation, vascular endothelial injury and the state of blood hypercoagulability. In this case, the favoring factor could be right-sided lymphadenopathy of the jugular vein which compresses the jugular vein and thus slows down the venous circulation. Even with simple suspicion of thrombosis, it is necessary to initiate heparinization of the patient. Our patient also benefited from low molecular weight heparin of the enoxaparin type (Lovenox 0.6UI twice daily for 3 days), an anti vitamin $\mathrm{K}$ genus acenocoumarol (Sintrom $4 \mathrm{mg} /$ day) with two INRs in the therapeutic range 2, 66 and 2.70. In the 
thromboembolic venous patient the target INR is between 2 and 3. Amoxicillin associated with clavulanic acid $1 \mathrm{~g}$ twice daily which is justified by leukocytosis. [5], hospitalization, the evolution was favorable with the complete disappearance of clinical signs and lymphadenopathy.

\section{Conclusion}

Jugular vein thrombosis is a rare variety of unusual localization of venous thromboembolic disease. It must be suspected in the presence of a painful cervical tummy and confirmed by Magnetic resonance imaging or to scan or ultrasound. The anticoagulant treatment should be instituted as soon as possible to avoid the formidable complication of pulmonary embolism.

\section{Conflicts of Interest}

The authors declare no conflicts of interest regarding the publication of this paper.

\section{References}

[1] Hergli, N., Hraiech, S., Hergli, W., Abdelati, S., Zemni, J. and Bougzala, E. (2005) Thrombosis of the Internal Jugulian Vein and Hyperhomocysteinemia. About a Case.

[2] Skouri, W., et al. (2017) Venous Thrombosis of an Unusual Seat in a Department of Internal Medicine.

[3] Haddad, S., El Euch, M., Mahfoudhi, M., Oueslati, O., Turki, S. and Ben, A. (2016) Venous Thromboses in a Department of Internal Medicine: About 58 Cases.

[4] Rachdi, E., et al. (2017) Thromboembolic Disease: What Is the Importance of Transient Risk Factors?

[5] Bartella, A., Lübbers, H.-T., Schuknecht, B., Gander, T., Grätz, K.W. and Kruse, A.L. (2014) Thrombosis of the External Jugular Vein. Swiss Dental Journal, 124, 39-48. 